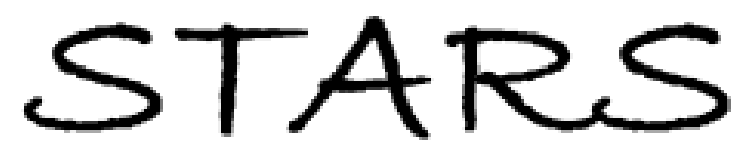

University of Central Florida

STARS

$1-1-2013$

\title{
Self-assembled palladium-organic composite nanofibers and their applications as a recyclable catalyst
}

\author{
Tanmay Bera \\ University of Central Florida \\ Jiyu Fang \\ University of Central Florida
}

Find similar works at: https://stars.library.ucf.edu/facultybib2010 University of Central Florida Libraries http://library.ucf.edu

This Article is brought to you for free and open access by the Faculty Bibliography at STARS. It has been accepted for inclusion in Faculty Bibliography 2010 s by an authorized administrator of STARS. For more information, please contact STARS@ucf.edu.

\section{Recommended Citation}

Bera, Tanmay and Fang, Jiyu, "Self-assembled palladium-organic composite nanofibers and their applications as a recyclable catalyst" (2013). Faculty Bibliography 2010s. 3699.

https://stars.library.ucf.edu/facultybib2010/3699

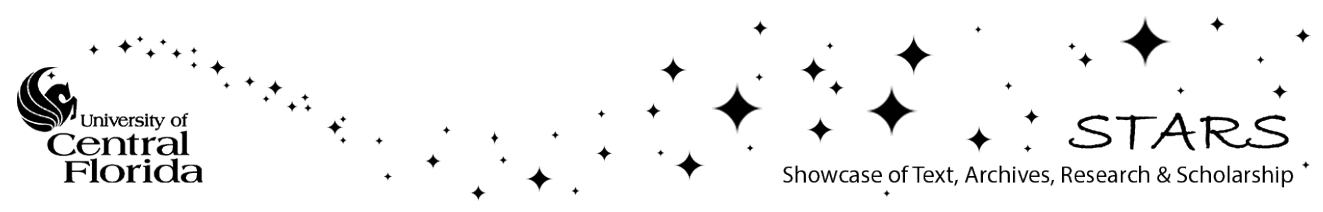




\title{
Self-assembled palladium-organic composite nanofibers and their applications as a recyclable catalyst
}

\author{
Tanmay Bera and Jiyu Fang*
}

We report the formation of palladium nanoparticle (PdNP)-lithocholic acid (LCA) composite nanofibers by

Received 18th April 2013

Accepted 6th September 2013

the self-assembly of $\mathrm{Pd}^{2+}$-coordinated LCA molecules and an ex situ reduction. The ex situ reduction by sodium borohydride or ascorbic acid produces the composite nanofibers with PdNPs grown on the surface of LCA nanofibers. The catalytic activity of the PdNP-LCA composite nanofibers in the decolourization of 4-phenyl azo benzoic acid is investigated. We find that the composite nanofibers can be recycled without significantly losing their catalytic activities.

www.rsc.org/advances

\section{Introduction}

Over the last decades, tremendous progress has been made in the synthesis of noble metal nanoparticles due to their applications as efficient nanocatalysts for chemical production, energy processes, and pollution controls. ${ }^{1-3}$ It is known that metal nanoparticles are thermodynamically unstable and tend to aggregate, which leads to the reduction of their catalytic activities. To prevent their aggregations, surfactants are often used as a stabilizing agent in the synthesis of metal nanoparticles. ${ }^{\mathbf{4}-13}$ However, the recyclability of metal nanoparticles still remains to be a challenge. To achieve the satisfactory recyclability, great efforts have been made in synthesizing onedimensional (1D) metal nanostructures ${ }^{14-17}$ or constructing metal nanoparticles on polymer nanofibers. ${ }^{\mathbf{1 8 - 2 1}}$

It has been shown that a variety of amphiphilic molecules are able to self-assemble into 1D supramolecular nanostructures with well-defined sizes and morphologies in solution. ${ }^{22-24}$ Significant progress has been made in using these self-assembled 1D supramolecular nanostructures such as fibers, ribbons and tubes as organized templates for growing and patterning inorganic nanoparticles. ${ }^{25-33}$ Recently, the selfassembly of metal ion-coordinated lipids has been proven to be a simple method for synthesizing composite nanotubes. ${ }^{34-36} \mathrm{Cu}$ ion- and Ni ion-coordinated lipid nanotubes have been proven to effective $1 \mathrm{D}$ catalysts for the oxidation of organic compounds. ${ }^{35,36}$ Due to the high aspect ratio of these $\mathrm{Cu}$ and $\mathrm{Ni}$ ion-coordinated lipid nanotubes, they can be easily separated from reaction solutions and show good recyclability.

Bile acids are important biological surfactants which are synthesized by the liver from the enzymatic catabolism of cholesterol. Unlike more traditionally amphiphilic surfactants

Department of Materials Science and Engineering, University of Central Florida, Orlando, Florida 32816, USA. E-mail: Jfang@mail.ucf.edu; Fax: +1-407-882-1462; Tel: $+1-407-882-1182$ with a hydrophilic head group bonded to a linear, flexible, hydrocarbon tail, bile acids have a large, rigid, quasi-planar steroid ring system with a hydrophilic $\alpha$ face and a hydrophobic $\beta$ face. It has been shown that amphipathic bile acids can selfassemble in aqueous solution into fibers, ${ }^{37}$ helical ribbons, ${ }^{38-40}$ and tubes ${ }^{41-49}$ depending on the experimental conditions under which self-assembly occurs. In a previous publication, ${ }^{47}$ we reported the formation of composite nanotubes by the selfassembly of $\mathrm{Cd}^{2+}$-coordinated lithocholic acid (LCA) in the presence of thioacetamide as a reducing agent. The formation of CdS nanoparticles in the tube walls makes the LCA nanotubes highly fluorescent. In this paper, we report the synthesis of PdNP-LCA composite nanofibers by the self-assembly of $\mathrm{Pd}^{2+}$-coordinated LCA molecules and an ex situ reduction approach. The ex situ reduction by sodium borohydride or ascorbic acid produces the composite nanofibers with the PdNPs grown on the surface of the LCA nanofibers. We show that the PdNP-LCA composite nanofibers can be used as a recyclable nanocatalyst for the effective decolourization of 4-phenyl azo benzoic acid in aqueous solution.

\section{Experimental section}

Lithocholic acid (LCA), 4-phenyl azo benzoic acid, ammonium hydroxide $\left(\mathrm{NH}_{4} \mathrm{OH}\right)$, palladium chloride $\left(\mathrm{PdCl}_{2}\right)$, ascorbic acid (AA), and sodium borohydride $\left(\mathrm{NaBH}_{4}\right)$ were purchased from Sigma-Aldrich. $\mathrm{Pd}^{2+}\left(\mathrm{NH}_{4} \mathrm{OH}\right)_{6} \mathrm{Cl}_{2}$ used as a precursor for the synthesis of PdNPs was prepared by dissolving $\mathrm{PdCl}_{2}$ in $\mathrm{NH}_{4} \mathrm{OH}$ aqueous solution. $\mathrm{NaBH}_{4}$ and $\mathrm{AA}$ were used as reducing agents. Water used in our experiments was purified with Easypure II system (18 $\mathrm{M} \Omega \mathrm{cm}, \mathrm{pH}$ 5.7). Carbon-coated copper grids were purchased from Electron Microscopy Science.

Transmission electron microscopy (TEM) was performed with a JEOL1011-EM microscope operating at an acceleration voltage of $100 \mathrm{kV}$. Ultraviolet-visible (UV-vis) spectra were recorded using a Cary 300 spectrophotometer. X-ray diffraction 
(XRD) measurements were carried out with a Rigaku D/max diffractometer with CuKa radiation $(\lambda=1.542 \AA)$ operated at $40 \mathrm{kV}$ and $30 \mathrm{~mA}$. Fourier transform infrared (FT-IR) spectra were recorded with a PerkinElmer 100 spectrometer equipped with a DTGS KBr detector.

\section{Results and discussion}

Fig. 1 shows the chemical structures of LCA and 4-phenyl azo benzoic acid. LCA is a secondary bile acid having a nearly planar hydrophobic steroid nucleus. The $\mathrm{COOH}$ group is linked to the steroid nucleus through a short alkyl chain. The deprotonated LCA molecules provides negatively charged $\mathrm{COO}^{-}$groups to coordinate with positively charged $\mathrm{Pd}^{2+}$ ions. In our experiments, $30 \mathrm{mg}$ of LCA was dissolved in $4 \mathrm{~mL}$ of $0.01 \mathrm{M}$ $\mathrm{NH}_{4} \mathrm{OH}$ aqueous solution at $\mathrm{pH}$ 11.0. The pre-prepared $\mathrm{Pd}^{2+}\left(\mathrm{NH}_{4} \mathrm{OH}\right)_{6} \mathrm{Cl}_{2}$ solution was added into $20 \mathrm{mM}$ LCA solution, in which the concentration of $\mathrm{Pd}^{2+}$ ions was varied from $0.1 \mathrm{mM}$ to $1 \mathrm{mM}$. The mixed solution was stirred for $60 \mathrm{~min}$ and then sealed in a glass vial at room temperature. After being aged for a week, the solution turns into milky color, suggesting that the self-assembly of $\mathrm{Pd}^{2+}$-coordinated LCA molecules takes place. Fig. 2a shows a typical TEM image of self-assembled nanofibers of $\mathrm{Pd}^{2+}$-coordinated LCA molecules, in which the self-assembled nanofibers was dried on a carbon-coated copper TEM grid. As can be seen in Fig. 2a, the $\mathrm{Pd}^{2+}$-coordinated LCA nanofibers are over $100 \mu \mathrm{m}$ long. Although the diameter of the nanofibers varies from one to another in the range from $70 \mathrm{~nm}$ to $180 \mathrm{~nm}$, individual nanofibers have a uniform diameter with a smooth surface. Fig. 2b shows the FT-IR spectra of the $\mathrm{Pd}^{2+}$-coordinated LCA nanofibers. The stretching of both the $\mathrm{COO}^{-}$group and the $\mathrm{COOH}$ group is observed. The absorption peak at $1748 \mathrm{~cm}^{-1}$ associates with the non-associated $\mathrm{COOH}$ groups. ${ }^{50}$ The absorption peaks at $1566 \mathrm{~cm}^{-1}$ and $1379 \mathrm{~cm}^{-1}$ represent the asymmetric and symmetric stretching of the $\mathrm{COO}^{-}$groups, respectively. The large separation between the asymmetric and symmetric stretching frequencies of the $\mathrm{COO}^{-}$groups suggests the coordination of $\mathrm{Pd}^{2+}$ ions to the $\mathrm{COO}^{-}$groups. ${ }^{51,52}$

After addition of $400 \mu \mathrm{L}$ of $10 \mathrm{mM} \mathrm{NaBH}_{4}$ aqueous solution, the milky-like nanofiber solution quickly turns into grayish color, suggesting the formation of PdNPs. Fig. 3a shows a typical TEM image of self-assembled PdNP-LCA composite nanofibers formed at the $\mathrm{Pd}^{2+}$ concentration of $0.2 \mathrm{mM}$. The formation of PdNPs with diameters of 25-30 $\mathrm{nm}$ on the LCA nanofibers is clearly visible in the TEM image. It is likely that

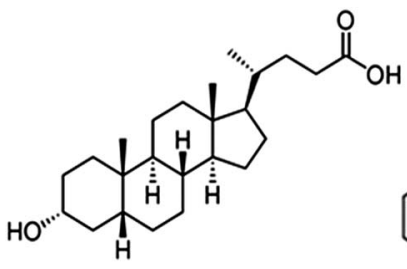

(a)

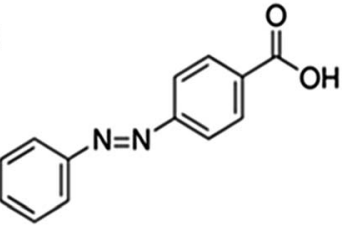

(b)
Fig. 1 Chemical structures of lithocholic acid (a) and 4-phenyl azo benzoic acid (b).
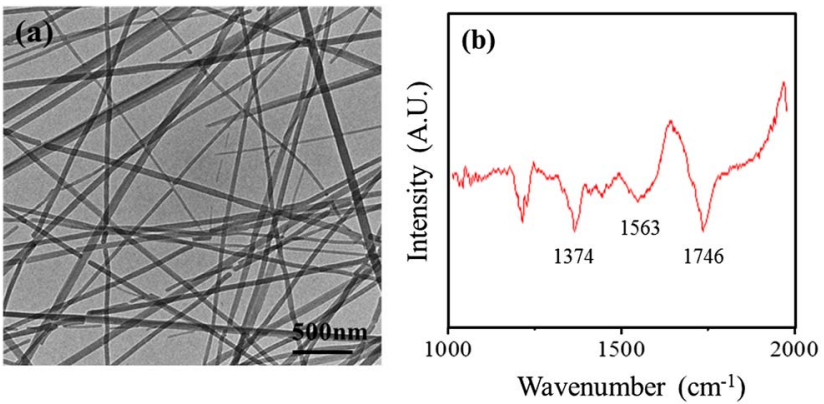

Fig. 2 (a) TEM image and (b) FT-IR spectrum of self-assembled $\mathrm{Pd}^{2+}$-coordinated LCA nanofibers formed at the $\mathrm{Pd}^{2+}$ ion concentration of $0.2 \mathrm{mM}$ before the reduction.

the coordinated $\mathrm{Pd}^{2+}$ ions exposed at the surface of the nanofibers are reduced by $\mathrm{NaBH}_{4}$ to form Pd nuclei and then grow into PdNPs. As the increase of $\mathrm{Pd}^{2+}$ concentrations, the number of the PdNPs grown on the surface of the nanofibers increases. The formation of clustered PdNPs on the surface of the nanofibers is observed when the $\mathrm{Pd}^{2+}$ concentration increases to $1 \mathrm{mM}$ (Fig. 3b). The size of the clustered PdNPs is in the range from $80 \mathrm{~nm}$ to $120 \mathrm{~nm}$. The X-ray diffraction pattern of the PdNP-LCA composite nanofibers show five characteristic peaks (Fig. 4), corresponding to the scattering from the (111), (200), (220), (311), and (222) planes of a face centered cubic Pd crystal structure.

The catalytic activity of PdNP-LCA composite nanofibers was evaluated in the decolourization of 4-phenyl azo benzoic acid. In our experiments, all the PdNP-LCA composite nanofibers were formed at the same LCA concentration $(20 \mathrm{mM})$ and then purified by centrifugation to remove excess LCA and free PdNPs. For the catalytic experiments discussed below, $25 \mu \mathrm{L}$ PdNP-LCA nanofiber solution was used. Since the concentration of LCA used for the self-assembly of composite nanotubes was kept to be constant, the amount of the composite nanofibers used for each case is roughly the same. When $25 \mu \mathrm{L}$ of $10 \mathrm{mM} \mathrm{NaBH}_{4}$ solution was added into $1 \mathrm{~mL}$ of $1 \mathrm{mM}$ 4-phenyl azo benzoic acid solution, there was no color change observed, suggesting that the decolourization proceeded at a very slow rate without catalysts. However, the addition of $25 \mu \mathrm{L}$ PdNP-LCA nanofiber solution could cause the ultimate fading of the orange color of
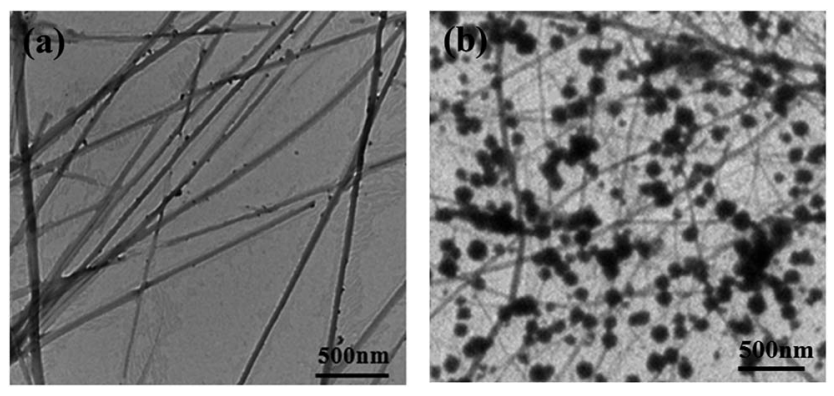

Fig. 3 TEM images of self-assembled $\mathrm{Pd}^{2+}$-coordinated LCA nanofibers formed at the $\mathrm{Pd}^{2+}$ ion concentration of $0.2 \mathrm{mM}(\mathrm{a})$ and $1 \mathrm{mM}(\mathrm{b})$ after the reduction by $\mathrm{NaBH}_{4}$. 


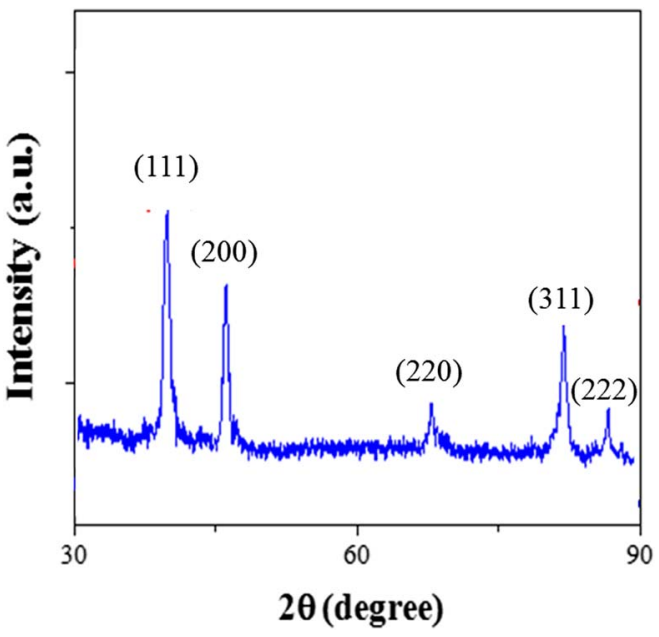

Fig. 4 X-ray diffraction pattern of self-assembled PdNP-LCA composite nanofibers formed at the $\mathrm{Pd}^{2+}$ ion concentration of $1 \mathrm{mM}$.

4-phenyl azo benzoic acid solution, suggesting that the PdNPLCA composite nanofibers were able to effectively catalyze the reduction of the $-\mathrm{N}=\mathrm{N}$ - bond of the azo benzoic acid in the presence of $\mathrm{NaBH}_{4}$.

To quantitatively evaluate the catalytic activity of PdNP-LCA composite nanofibers formed at different conditions, we study the kinetics of the decolourization reaction by monitoring the reaction solution with UV-vis absorption spectroscopy as a function of time at room temperature. Fig. 5a shows the timeresolve UV-vis absorption spectra of the decolourization of 4-phenyl azo benzoic acid by the PdNP-LCA composite nanofibers synthesized in the $\mathrm{Pd}^{2+}$ concentration of $1 \mathrm{mM}$, where $\mathrm{NaBH}_{4}$ was used as a reducing agent. The UV-vis absorption spectra were recorded in every $1 \mathrm{~min}$. In the absence of the PdNP-LCA nanofibers, 4-phenyl azo benzoic acid solution shows a characteristic absorption peak at $430 \mathrm{~nm}$ arising from the $-\mathrm{N}=\mathrm{N}-$ bond of the azo benzoic acid. As can be seen in Fig. 5a, the adsorption peak quickly drops over time after the addition of $25 \mu \mathrm{L}$ PdNP-LCA nanofiber solution into $1 \mathrm{~mL}$ of $1 \mathrm{mM}$ 4-phenyl azo benzoic acid solution, suggesting that the nanofibers are capable of catalyzing the reduction of the $-\mathrm{N}=$ $\mathrm{N}$ - bond. After $10 \mathrm{~min}$, the absorption peak at $430 \mathrm{~nm}$ disappears. Thus, the intensity of the absorption peak was used to quantitatively evaluate the catalytic performance of the PdNPLCA composite nanofibers. Fig. $5 \mathrm{~b}$ shows the plots of $\ln \left(A_{t} / A_{0}\right)$ versus reaction time $t$ for the decolourization of 4-phenyl azo benzoic acid by the PdNP-LCA composite nanofibers, where $A_{t}$ and $A_{0}$ is the intensity of absorption peaks at time $t$ and 0 , respectively. The linear relationship shown in Fig. 5b suggests that the decolourization process by the PdNP-LCA composite nanofibers follows pseudo-first-order kinetics. Since the concentration of $\mathrm{NaBH}_{4}$ is high, we expect that it remains essentially constant during the reaction. Thus, the pseudo-firstorder kinetics could be used to evaluate the catalytic rate of the PdNP-LCA composite nanofibers in the decolourization of 4-phenyl azo benzoic acid. The rate constant calculated from the slope of the plots shown in Fig. $5 \mathrm{~b}$ is $9.24 \times 10^{-4} \mathrm{~s}^{-1}$. We also carried out the catalytic experiment by using AA as a reducing agent. Where $25 \mu \mathrm{L}$ of $10 \mathrm{mM}$ AA solution was added into $1 \mathrm{~mL}$ of $1 \mathrm{mM}$ 4-phenyl azo benzoic acid solution, followed by the addition of $25 \mu \mathrm{L}$ PdNP-LCA nanofiber solution. The pseudo-first-order kinetics is also observed (Fig. 5c). In this case, the calculated rate constant is $3.91 \times 10^{-4} \mathrm{~s}^{-1}$. The reduced rate constant is due to the fact that $\mathrm{AA}$ is a weaker reducing agent, compared to $\mathrm{NaBH}_{4}$.

Furthermore, we study the catalytic performance of the PdNP-LCA nanofibers synthesized at different $\mathrm{Pd}^{2+}$ concentrations. $25 \mu \mathrm{L}$ of the PdNP-LCA nanofiber solutions was added to $1 \mathrm{~mL}$ of $1 \mathrm{mM}$ 4-phenyl azo benzoic acid solution in the presence of $\mathrm{NaBH}_{4}$ as a reducing agent. Fig. 6a shows the plots of $\ln \left(A_{t} / A_{0}\right)$ versus reaction time $t$ for the decolourization of 4-phenyl azo benzoic acid by the PdNP-LCA composite nanofibers formed at different $\mathrm{Pd}^{2+}$ concentrations. As can be seen, all the decolourization processes show pseudo-first-order kinetics. But, the rate constant decreases from $9.24 \times 10^{-4} \mathrm{~s}^{-1}$ to $0.6 \times 10^{-4} \mathrm{~s}^{-1}$ when the $\mathrm{Pd}^{2+}$ concentration used for forming the PdNP-LCA composite nanofibers decreases from $1 \mathrm{mM}$ to $0.1 \mathrm{mM}$ (Fig. 6b). Since the amount of the composite nanofibers is roughly the same, the decrease in the rate constant is a result of the decrease of the number of PdNPs on the surface of the nanofibers (see Fig. 3).

The recycling ability is an important property to estimate the catalytic performance of a catalyst. In our experiments, PdNPLCA composite nanofibers were separated from the reaction solution by simple centrifugation after each reaction cycle. Fig. 7a shows a typical TEM image of the PdNP-LCA nanofibers
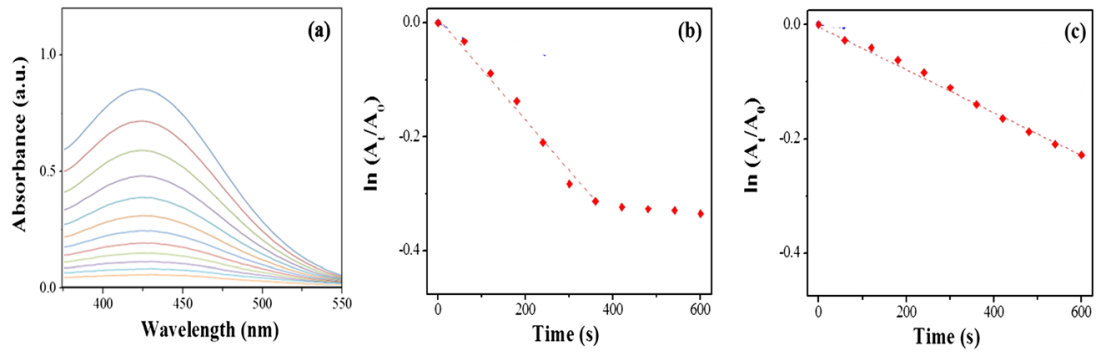

Fig. 5 (a) Time-resolved UV-vis absorption spectra for the decolourization of 4-phenyl azo benzoic acid by the self-assembled PdNP-LCA composite nanofibers in the presence of $\mathrm{NaBH}_{4}$. The spectra were recorded every 1 min. Plots of $\ln \left(A / A_{0}\right)$ versus time for the decolourization of 4-phenyl azo benzoic acid by the PdNP-LCA composite nanofibers in the presence of $\mathrm{NaBH}_{4}$ (b) and $\mathrm{AA}$ (c). 

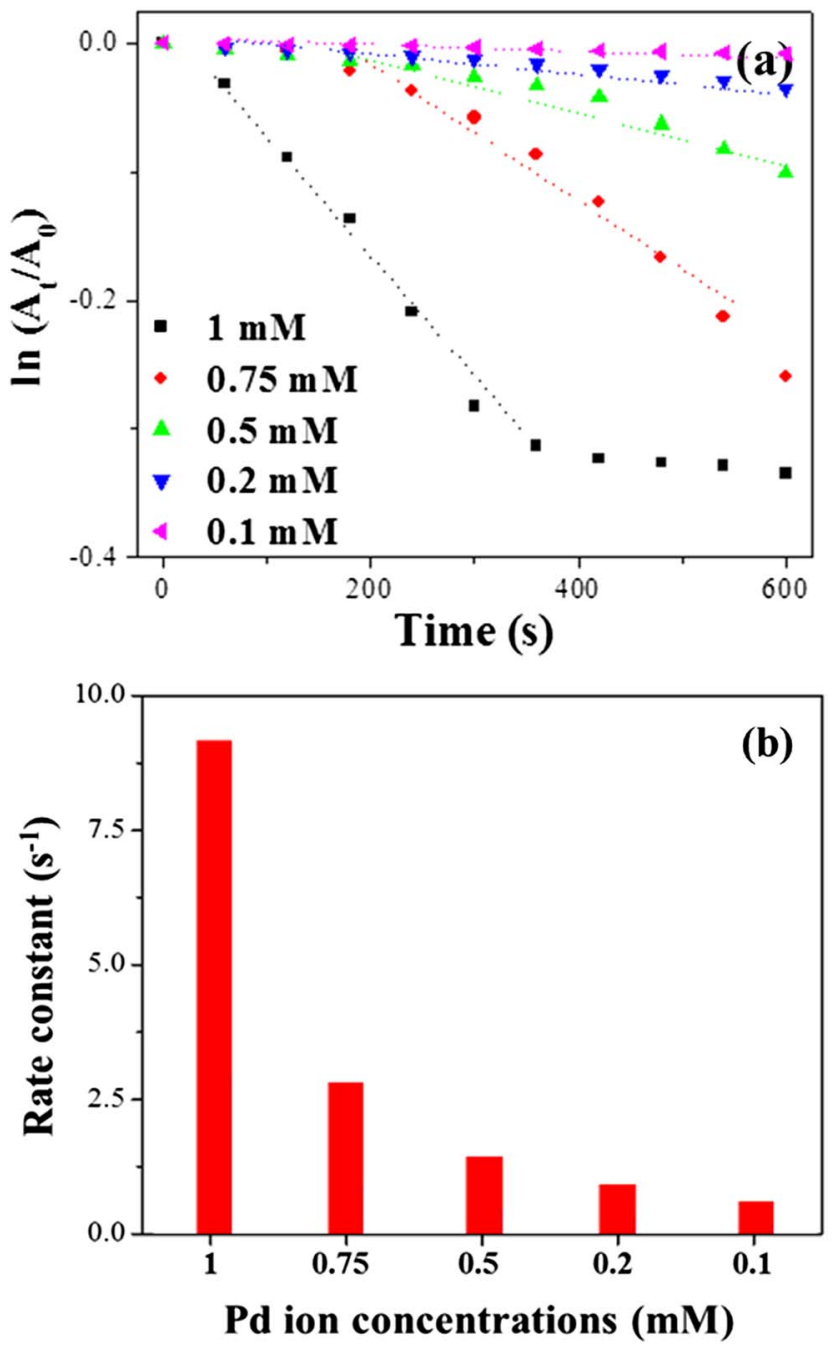

Fig. 6 (a) Plots of $\ln \left(A / A_{0}\right)$ versus time for the decolourization of 4-phenyl azo benzoic acid by the self-assembled PdNP-LCA composite nanofibers synthesized in different $\mathrm{Pd}^{2+}$ concentrations, in which $\mathrm{NaBH}_{4}$ was used as a reducing agent. (b) Rate constants versus $\mathrm{Pd}^{2+}$ concentrations.

synthesized at the $\mathrm{Pd}^{2+}$ concentration of $1 \mathrm{mM}$ after the first reaction cycle. There is no apparently structural change in the size of clustered PdNPs and LCA nanofibers observed. Fig. 7b shows the plots of $\ln \left(A_{t} / A_{0}\right)$ versus reaction time $t$ for the decolourization of 4-phenyl azo benzoic acid by the PdNP-LCA composite nanofibers for four recycling tests, where $\mathrm{NaBH}_{4}$ is used as a reducing agent. As can be seen in Fig. 7b, the decolourization process of each recycles shows pseudo-first-order kinetics. There is a relatively large decrease in the rate constant from $9.24 \times 10^{-4} \mathrm{~s}^{-1}$ to $6.02 \times 10^{-4} \mathrm{~s}^{-1}$ from the first recycling to the second recycling (Fig. 7c). This might be a result of losing some short composite nanofibers and/or clustered PdNPs during the separation process. However, after the first recycling the rate constant of the decolourization of 4-phenyl azo benzoic acid by the PdNP-LCA composite nanofibers is found to be near the same (Fig. 7c), suggesting that the PdNP-LCA composite nanofibers can be used as a recyclable nanocatalyst for the effective decolourization of 4-phenyl azo benzoic acid in aqueous solution.

If the concentration of LCA is reduced to from $20 \mathrm{mM}$ to $0.3 \mathrm{mM}$, there is no PdNP-LCA composite nanofiber observed. Instead, we observe the formation of PdNP-LCA nanoparticles with a diameter of 20-30 nm (Fig. 8a). Again, X-ray diffraction of PdNP-LCA composite nanoparticles shows five characteristic peaks from the scattering of the (111), (200), (220), (311), and (222) planes of a face centered cubic Pd crystal structure. For studying the catalytic performance of PdNP-LCA nanoparticles, we added $25 \mu \mathrm{L}$ of PdNP-LCA nanoparticle solution into $1 \mathrm{~mL}$ of $1 \mathrm{mM}$ 4-phenyl azo benzoic acid solution in the presence of $\mathrm{NaBH}_{4}$. After each reaction cycle, the PdNP-LCA composite nanoparticles were separated from the reaction solution by centrifugation under the same condition used for the separation of PdNP-LCA composite nanofibers. Fig. 8b shows the plots of $\ln \left(A_{t} / A_{0}\right)$ versus reaction time $t$ for the decolourization of 4-phenyl azo benzoic acid by the PdNP-LCA composite nanoparticles for four recyclings. The rate constant drops significantly from $27.6 \times 10^{-4} \mathrm{~s}^{-1}$ to $0.87 \times 10^{-4} \mathrm{~s}^{-1}$. At the same time, we note that the PdNP-LCA composite nanoparticles are more difficult to be separated from the solution than the PdNPLCA composite nanofibers by sedimentation under the same centrifugation condition. Thus, the significant decrease of the rate constant is likely a result of the decrease of the number of the PdNP-LCA composite nanoparticles used for the recycling. In addition, we can not completely rule out the possibility of leaching some ions from the PdNP-LCA composite nanoparticles during the reaction, which has been reported in
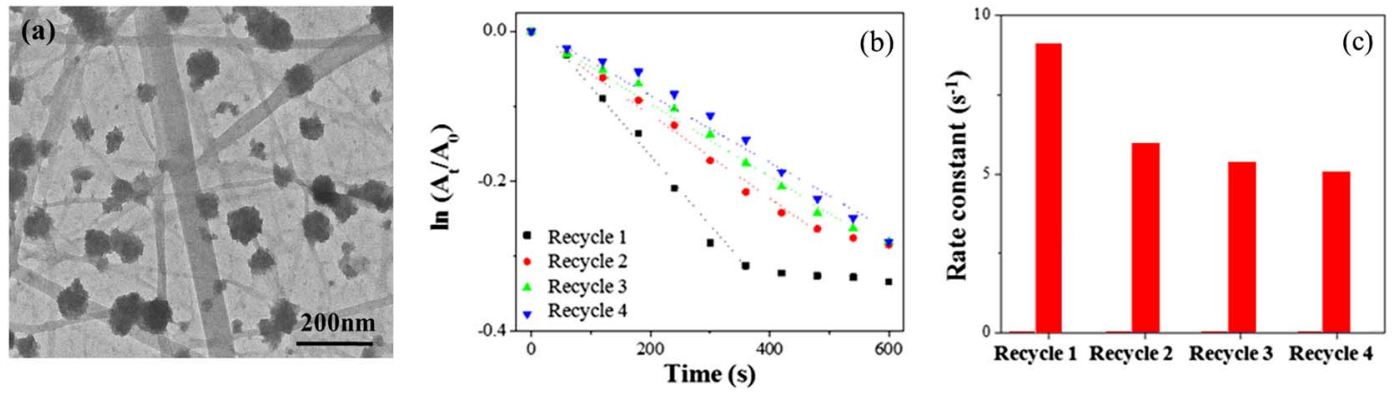

Fig. 7 (a) TEM image of PdNP-LCA composite nanofibers formed at the $\mathrm{Pd}^{2+}$ ion concentration of $1 \mathrm{mM}$ after the first reaction cycle. (b) Plots of $\mathrm{In}\left(A / A_{0}\right)$ versus time for the decolourization of 4-phenyl azo benzoic acid by PdNP-LCA composite nanofibers for four recycles, in which $\mathrm{NaBH}_{4}$ was used as a reducing agent. (c) Rate constants versus recycles. 

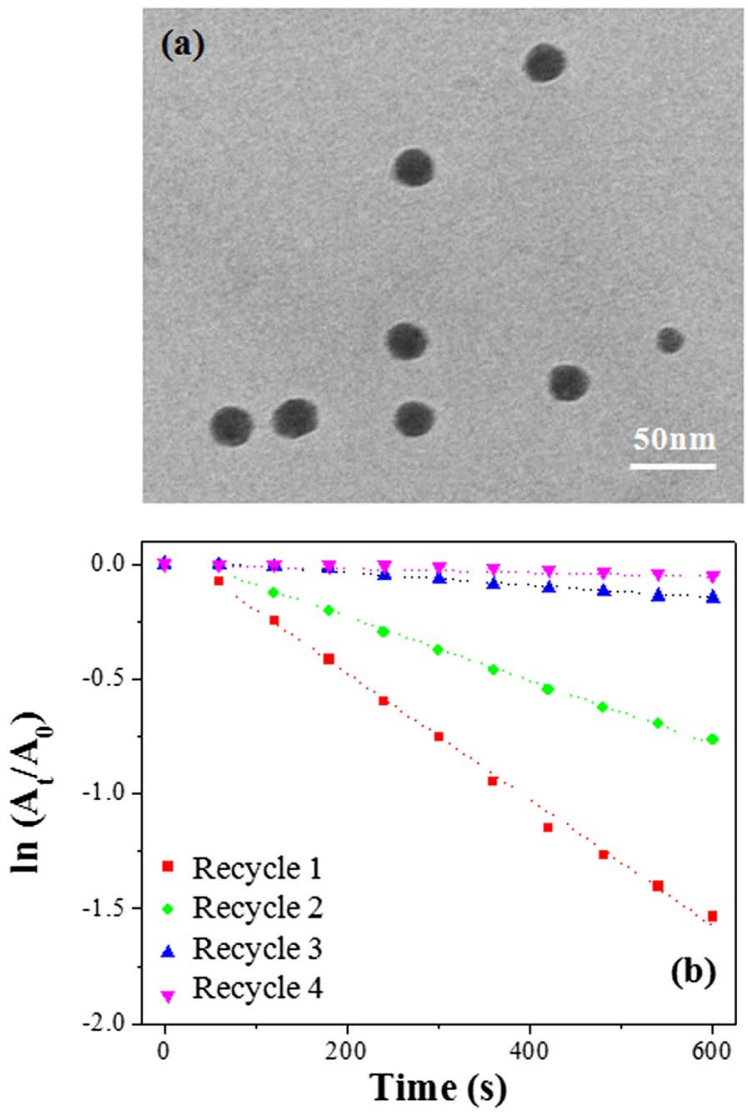

Fig. 8 (a) TEM image of PdNP-LCA nanoparticles. (b) Plots of $\ln \left(A / A_{0}\right)$ versus time for the decolourization of 4-phenyl azo benzoic acid by PdNP-LCA composite nanoparticles for four recycles.

PdNP-catalyzed Heck and Suzuki reactions..$^{53,54}$ It has been shown that the leaching of ions from PdNPs can lead to the change of their sizes and shapes. ${ }^{54}$ However, due to the size variations of the initially formed PdNP-LCA composite nanoparticles, it is difficult to verify their small size changes after the reaction.

In summary, we report the formation of PdNP-LCA composite nanofibers by the self-assembly of $\mathrm{Pd}^{2+}$-coordinated LCA molecules and an ex situ reduction approach. The PdNPLCA composite nanofibers show good catalytic activity in the decolourization of 4-phenyl azo benzoic acid. Due to the high aspect ratio, the PdNP-LCA composite nanofiber catalysts can be easily separated from reaction solution by centrifugation. We demonstrate that the PdNP-LCA composite nanofiber catalysts can be reused without significantly losing their catalytic activities.

\section{Acknowledgements}

This work was supported by the National Science Foundation (CBET 0855322).

\section{References}

1 A. Roucoux, J. Schulz and H. Patin, Chem. Rev., 2002, 102, 3757-3778.
2 D. Astruc, F. Lu and J. R. Aranzaes, Angew. Chem., Int. Ed., 2005, 44, 7852-7872.

3 M. J. Climent, A. Corma and S. Iborra, Chem. Rev., 2011, 111, 1072-1133.

4 S. Klingelhöfer, W. Heitz, A. Greiner, S. Oestreich, S. Förster and M. Antonietti, J. Am. Chem. Soc., 1997, 119, 10116-10120.

5 A. Henglein, B. G. Ershov and M. Malow, J. Phys. Chem., 1995, 99, 14129-14136.

6 H. Ohde, C. M. Wai, H. Kim, J. Kim and M. Ohde, J. Am. Chem. Soc., 2002, 124, 4540-4541.

7 B. H. Yoon, H. Kim and C. M. Wai, Chem. Commun., 2003, 1040-1041.

8 P. Meric, K. M. Yu and S. C. Tsang, Langmuir, 2004, 20, 85378545.

9 J. Liu, J. Alvarez, W. Ong, E. Roman and A. E. Kaifer, Langmuir, 2001, 17, 6762-6764.

10 C. L. Lee, C. C. Wan and Y. Y. Wang, Adv. Funct. Mater., 2001, 11, 344-347.

11 F. Ibanez and F. P. Zamborini, J. Am. Chem. Soc., 2008, 130, 622-633.

12 E. Sadeghmoghaddam, C. Lam, D. Choi and Y. S. Shon, J. Mater. Chem., 2011, 21, 307-312.

13 M. Moreno, F. J. Ibañez, J. B. Jasinski and F. P. Zamborini, J. Am. Chem. Soc., 2011, 133, 4389-4397.

14 Z. Chen, M. Waje, W. Li and Y. Yan, Angew. Chem., Int. Ed., 2007, 46, 4060-4063.

15 H. J. Zhou, W. P. Zhou, R. R. Adzic and S. S. Wong, J. Phys. Chem. C, 2009, 113, 5460-5466.

16 B. Lim and Y. N. Xia, Angew. Chem., Int. Ed., 2011, 50, 76-85.

17 Z. Niu, D. Wang, R. Yu, Q. Peng and Y. Li, Chem. Sci., 2012, 3, 1925-1929.

18 M. M. Demir, M. A. Gulgun, Y. Z. Menceloglu, B. Erman, S. S. Abramchuk, E. E. Makhaeva, A. R. Khokhlov, V. G. Matveeva and M. G. Sulman, Macromolecules, 2004, 37, 1787-1792.

19 X. Fang, H. Ma, S. Xiao, M. Shen, R. Guo, X. Cao and X. Shi, J. Mater. Chem., 2011, 21, 4493-4501.

20 H. Ma, Y. Huang, M. Shen, D. Hu, H. Yang, M. Zhu, S. Yang and X. Shi, RSC Adv., 2013, 3, 6455-6465.

21 M. Cao, L. Zhou, X. Xu, S. Cheng, J.-L. Yao and L.-J. Fan, J. Mater. Chem. A, 2013, 1, 8942-8949.

22 M. S. Spector, J. V. Selinger and J. M. Schnur, MaterialsChirality: Vol. 24 of Topics in Stereochemistry: Chiral molecular self-assembly, ed. M. M. Green, R. J. M. Nolte and E. W. Meijer, Wiley, Hoboken, 2003.

23 T. Shimizu, M. Masuda and H. Minamikawa, Chem. Rev., 2005, 105, 1401-1443.

24 J. F. Fang, J. Mater. Chem., 2007, 17, 3479-3484.

25 S. L. Burkett and S. Mann, Chem. Commun., 1996, 321-322.

26 Y. M. Lvov, R. R. Price, J. V. Selinger, A. Singh, M. S. Spector and J. M. Schnur, Langmuir, 2000, 16, 5932-5935.

27 A. Banerjee, L. Yu and H. Matsui, Proc. Natl. Acad. Sci. U. S. A., 2003, 100, 14678-14682.

28 A. Banerjee, L. Yu and H. Matsui, Nano Lett., 2003, 3, 283287.

29 R. Takahashi and T. Ishiwatari, Chem. Commun., 2004, 14061407. 
30 J. H. Jung, J. A. Rim, S. J. Lee and S. S. Lee, Chem. Commun., 2005, 468-470.

31 P. Terech, N. M. Sangeetha, S. Bhat, J. J. Allegraud and E. Buhler, Soft Matter, 2006, 2, 517-522.

32 C. Park, M. S. Im, S. Lee, J. Lim and C. Kim, Angew. Chem., Int. Ed., 2008, 47, 9922-9926.

33 Y. Qiao, Y. Lin, Y. Wang, Z. Yang, J. Liu, J. Zhou, Y. Yan and J. Huang, Nano Lett., 2009, 9, 4500-4504.

34 C. He, Y. Shimizu, N. Koshizaki and T. Shimizu, Adv. Mater., 2007, 19, 1055-1058.

35 T. Chattopadhyay, M. Kogiso, M. Asakawa, T. Schimizu and M. Aoyagi, Catal. Commun., 2010, 12, 9-13.

36 T. Chattopadhyay, M. Kogiso, M. Aoyagi, H. Yui, M. Asakawa and T. Schimizu, Green Chem., 2011, 13, 1138-1140.

37 D. M. Blow, J. Am. Chem. Soc., 1960, 82, 3566-3572.

38 Y. V. Zastavker, N. Asherie, A. Lomakin, J. Pande, J. M. Donovan, J. M. Schnur and G. B. Benedek, Proc. Natl. Acad. Sci. U. S. A., 1999, 96, 7883-7887.

39 Y. Qiao, Y. Lin, Z. Yang, H. Chen, S. Zhang, Y. Yan and J. Huang, J. Phys. Chem. B, 2010, 114, 11725-11730.

40 P. Terech, S. K. P. Velu, P. Pernot and L. Wiegart, J. Phys. Chem. B, 2012, 116, 11344-11355.

$41 \mathrm{P}$. Terech and Y. Talmon, Langmuir, 2002, 18, 7240-7244.

42 N. Mangisi, C. Leggio, A. J. Jover, F. Meijide, N. V. Pavel, V. H. S. Tellini, J. V. Tato, R. G. Agostino and L. Galantini, Angew. Chem., Int. Ed., 2010, 49, 6604-6607.
43 A. Pal, H. Basit, S. Sen, V. K. Aswal and S. J. Bhattacharya, J. Mater. Chem., 2009, 19, 4325-4334.

44 X. Zhang, J. Zou, K. Tamhane, F. Kobzeff and J. Y. Fang, Small, 2010, 6, 217-220.

45 K. Tamhane, X. Zhang, J. Zou and J. Y. Fang, Soft Matter, 2010, 6, 1224-1228.

46 S. Song, L. Feng, A. Song and J. Hao, J. Phys. Chem. B, 2012, 116, 12850-12856.

47 X. Zhang, M. Mathew, A. J. Gesquiere and J. Y. Fang, J. Mater. Chem., 2010, 20, 3716-3721.

48 X. Zhang, T. Bera, W. Liang and J. Y. Fang, J. Phys. Chem. B, 2011, 115, 1445.

49 L. Schefer, A. Sachez-Ferrer, J. Adamcik and R. Mezzenga, Langmuir, 2012, 28, 5999-6005.

50 A. M. Lichkus, P. C. Painter and M. M. Coleman, Macromolecules, 1988, 21, 2636-2641.

51 P. J. Thistlethwaite and M. S. Hook, Langmuir, 2000, 16, 4993-4998.

52 A. D. Buckland, C. H. Rochester and S. A. Topham, J. Chem. Soc., Faraday Trans. 1, 1980, 76, 302313.

53 A. V. Gaikwad, A. Holuigue, M. B. Thathagar, J. E. ten Elshof and G. Rothenberg, Chem.-Eur. J., 2007, 13, 69086913.

54 Z. Niu, Q. Peng, Z. Zhuang, W. He and Y. Li, Chem.-Eur. J., 2012, 18, 9813-9817. 